\title{
EDITORIAL
}

\section{Drug treatment of hypertension: implications of ALLHAT}

\section{B Williams}

Heart 2003;89:589-590

The largest ever study of antihypertensive treatment, the ALLHAT trial, has led to some controversial conclusions about the safety and efficacy of the "newer" antihypertensive agents the thiazide was "the winner". ${ }^{13}$ If the primary end point and all cause mortality are the same with all three drugs classes, how could this interpretation have arisen? The premise that chlorthalidone was "the winner" comes from analysis of secondary end points and assumptions about cost and thus "cost effectiveness". However, confidence in the robustness of the effectiveness data is essential before cost effectiveness can be considered.

There were differences in two secondary outcome measures in ALLHAT: stroke, and heart failure. The incidence of stroke was 15\% higher with lisinopril when compared to chlorthalidone, and the rate of heart failure was reported as 19\% higher with lisinopril and 38\% higher with amlodipine when compared to the diuretic. If this is true, then these findings would represent important differences in drug specific effects. However, there are some troubling inconsistencies in these findings that have more to do with the design of the trial than drug specific effects.

Blood pressure lowering is a powerful determinant of benefit in clinical trials, even more powerful than often recognised. ${ }^{4}$ Thus, even small differences in blood pressure between the treatment groups would have a powerful effect on outcome, especially for stroke. The ALLHAT population had a mean age of 67 years and 35\% were black. Older people and blacks respond less well to ACE inhibition and much better to diuretics with regard to blood pressure lowering. ${ }^{56}$ Thus, it was unsurprising that systolic blood pressure control was significantly lower in those receiving diuretics when compared to those receiving ACE inhibition throughout the trial. ${ }^{1}$ This was especially noticeable in blacks. The magnitude of this difference would be sufficient to account for the less effective outcome for lisinopril when compared to chlorthalidone for stroke protection. ${ }^{4}$

\section{CONTENTIOUS DATA REGARDING HEART FAILURE}

The data regarding heart failure is more contentious and has been a controversial end point in ALLHAT ever since the premature discontinuation of the doxazosin arm of the study for a reported excess of heart failure when compared to the diuretic. ${ }^{27}$ This again may have much to do with trial design. It is not widely recognised that $90 \%$ of patients randomised into ALLHAT were receiving antihypertensive treatment. The details of this baseline treatment have not been reported

Correspondence to: Dr Bryan Williams, Clinical Sciences Building, Leicester Royal Infirmary, PO Box 65, Leicester LE2 7LX, UK; bw17@le.ac.uk

\section{NO DIFFERENCE IN PRIMARY END POINT RATE}

At the end of the study, there was no difference in the rate of the primary end point when compa ing the reference drug chlorthalidone (11.5\%) with amlodipine (11.3\%) or with lisinopril (11.4\%). ${ }^{1}$ Moreover, there was no difference in the most important secondary end point-death. Compared with chlorthalidone, all cause mortality with lisinopril was identical and nonsignificantly $4 \%$ lower with amlodipine. Mindful of these outcomes, it was surprising that the investigators, the accompanying editorial in the $J A M A$, and the subsequent publicity, declared that
Abbreviations: $A C E$, angiotensin converting enzyme; ALLHAT, antihypertensive and lipid lowering treatment to prevent heart attack trial; CCB, calcium channel blocker 
and it is unclear if they are even available. This is very important because there was no "washout period" or placebo run-in. Patients were simply crossed over to their randomised medications. It is likely that many of this elderly cohort were receiving diuretic treatment at baseline and its cessation and subsequent crossover to an ACE inhibitor or CCB would be expected to unmask occult heart failure. Consistent with this possibility, the rates of heart failure diverge very early and then appear to remain parallel thereafter. Moreover, the expected higher mortality associated with clinical heart failure is inconsistent with the finding that all cause mortality rates are similar with all three drug classes and if anything lower with amlodipine! Heart failure is a difficult end point to validate in such a large clinical trial and in my view the findings of ALLHAT with regard to heart failure should continue to be viewed with caution. The overemphasis given to this end point by the report of the findings of ALLHAT in JAMA and the subsequent publicity distorted the balance of the overall conclusion, away from the primary end point. So much so that even the New York Times felt the need to subsequently publish a correction of its initial front page interpretation of the breaking news from ALLHAT.

What have we learnt from ALLHAT? We have gained reassurance that the longstanding recommendations of many hypertension societies in Europe are correct and that thiazide diuretics are the most effective initial treatment for lowering blood pressure in older people and should form the platform for drug therapy for hypertension in older people. We have learnt that epidemiologists can at times be more dangerous than the diseases they study and that the misguided publication of scare stories about increased risks of coronary heart disease, cancer, and gastrointestinal bleeding with CCBs has not been confirmed. There is no substitute for a large scale trial when defining treatment modalities to which massive numbers of the population will be exposed. Trials are as much a study of safety as they are of efficacy and the findings of ALLHAT in this regard are reassuring. Finally, we have learnt once again that most people will require more than one drug to control their blood pressure and that the debate about initial therapy is less important than defining the composition of the optimal therapeutic combination for the treatment of hypertension. $^{89}$

\section{REFERENCE}

1 The ALLHAT Officers and Coordinators for the ALLHAT Collaborative Research Group. Major outcomes in high-risk hypertensive patients randomized to angiotensin-converting enzyme inhibitor or calcium channel blocker vs diuretic: the antihypertensive and lipid lowering treatment to prevent heart attack trial (ALLHAT). JAMA 2002;288:2981-97.

2 The ALLHAT Officers and Co-ordinators for the ALLHAT Collaborative Research Group. Major cardiovascular events in hypertensive patients randomly assigned to doxazosin vs chlorthalidone: the antihypertensive and lipid lowering treatment to prevent heart attack trial (ALLHAT). JAMA 2000;283:1967-75.

3 Appel LJ. The verdict from ALLHAT - thiazide diuretics are the preferred initial therapy for hypertension. JAMA 2002;288:3039-41.

4 Prospective Studies Collaboration. Age-specific relevance of usual blood pressure to vascular mortality: a meta-analysis of individual data for one million adults in 61 prospective studies. Lancet 2002;360:1903-13.

5 Dickerson JE, Hingorani AD, Ashby M, et al. Optimisation of antihypertensive treatment by crossover rotation of four major classes. Lancet 1999;353:2008-13.

6 Brown MJ, Cruickshank JK, Dominiczak AF, et al. Better blood pressure control: how to combine drugs. J Hum Hypertension 2003;17:81-6.

7 Poulter NR, Williams B. Doxazosin for the management of hypertension: implications of the ALLHAT trial. Am J Hypertension 2001;14:1170-2.

8 Williams B. Drug treatment of hypertension. BM 2003;326:61-2.

9 Williams B. Treating hypertension: it is not how you start but where you end that matters. J Hypertension 2003;21:211-13.

\title{
ELECTRONIC PAGES
}

\author{
eHEART: www.heartinl.com
}

Thun

he following electronic only articles are published in conjunction with this issue of Heart.

\section{Novel use of an "insertable" loop recorder}

J R Gimbel

A patient with palpitations and suspected arrhythmia underwent Holter and external loop recorder monitoring. No arrhythmias were detected by these traditional monitoring methods. An insertable loop recorder (ILR) was placed on the patient's chest and used as an extended loop recorder. An arrhythmia was ultimately recorded by the externally placed ILR leading to appropriate treatment.

(Heart 2003;89:el8) www.heartjnl.com/cgi/content/full/89/ $6 / \mathrm{e} 18$
A 28 year old woman with ventricular tachycardia and an abnormal chest radiograph

M Heath, H Deubner, E J Stern, J E Cooke, E A Gill

A 28 year old white woman with no medical history presented to the emergency room with symptomatic non-sustained ventricular tachycardia. She was asymptomatic up to a few days before presentation. Her physical examination was essentially normal and hence did not help with the differential diagnosis of the problem. Bronchoscopic transbronchial biopsy led to the final diagnosis of cardiac sarcoidosis.

(Heart 2003;89:e19) www.heartjnl.com/cgi/content/full/89/ 6/e19 